\title{
Frozen state conditions for exponential consensus of time-varying cooperative nonlinear networks
}

\author{
Sabato Manfredi ${ }^{a}$ David Angeli ${ }^{b}$ \\ ${ }^{a}$ Department of Electrical Engineering and Information Technology, University of Naples Federico II, Italy and Control and \\ Power Group, Electrical and Electronic Engineering Department, Imperial College, London. Email: sabato.manfredi@unina.it \\ ${ }^{\mathrm{b}}$ Control and Power Group, Electrical and Electronic Engineering Department, Imperial College, London and Dip. di \\ Ingegneria dell'Informazione, University of Florence, Italy.Email: d.angeli@imperial.ac.uk
}

\begin{abstract}
In this paper we present new results on exponential consensus for continuous-time nonlinear time varying cooperative networks. We endow the well known assumption of integral connectivity proposed by Moreau with a remarkable additional feature of being frozen in state variables, making its direct verification more straightforward. Moreover, we give an estimate of the exponential rate of convergence towards the agreement space. Finally, the proposed results are validated for representative examples.
\end{abstract}

Key words: Monotone Systems, Consensus, Synchronization, Multi agent systems.

\section{Introduction}

Many complex systems of interest, both in nature and engineering, can be modelled as large collections of interacting subsystems. In recent years, the increased number of control applications involving formation flight, sensor networks, swarms, collective behavior of flocks, etc., has triggered a significative attention of the scientific community to the consensus, coordination and synchronization problems of multiple dynamical systems operating over a network (see e.g. [Murray, 2007] and references therein). One common feature of the above problems is to allow every agent to autonomously converge to a common agreement value about some variables of interest by only processing local information at the node and/or information received from neighbooring agents. Both from a theoretical and practical point of view it is of interest to qualify structural conditions on the network topology or individual agents dynamics which may guarantee asymptotic convergence to an agreement state. Although remarkable convergence results have been formulated for the discrete time (e.g. [Moreau, 2005]) herein we draw our attention to the problem of continuous time agreement. A noticeable result about minimal connectivity assumptions (integral

\footnotetext{
* A preliminary and partial version of the paper was accepted to the 2013 Conference on Decision and Control, Florence, Italy, 2013 [Manfredi \& Angeli, 2013]
}

connectivity) to guarantee convergence to agreement is given in [Moreau, 2004] in the case of time varying communication links: the existence of a spanning tree for the $\delta$-digraph is sufficient for network agreement. Recently several notions of connectivity have been formulated in the literature with the aim of ensuring asymptotic convergence to consensus (i.e. [Hendrickx \& Tsitsiklis, 2013], [Martin \& Girard, 2013]), [Cao et. al, 2011]). In [Cao et. al, 2011] the consensus is guaranteed for undirected networks (e.g. symmetric interactions) under the infinite integral connectivity condition: the "infinite integral graph" obtained by connecting $i$ to $j$ whenever $\int_{0}^{\infty} a_{i j}(\tau) d \tau=\infty$. In [Hendrickx \& Tsitsiklis, 2013] the consensus is achieved under this same assumption (renamed unbounded interactions graph) along with a condition of cut-balanced interactions. In [Martin \& Girard, 2013] the authors extend the continuous time result in [Hendrickx \& Tsitsiklis, 2013] under the assumption of infinite integral connectivity (therein named persistent connectivity) and replacing the cut-balanced interactions assumption by the weaker one of slow divergence of reciprocal weights: namely, the ratio of reciprocal weights is at worst slowly diverging to infinity. Additionally, an explicit bound on the convergence rate to the consensus is provided. By adopting a similar definition (namely a "persistent graph" obtained connecting $i$ to $j$ whenever $\int_{s}^{\infty} a_{i j}(\tau) d \tau=\infty$ for all $s \geq 0$ ), an alternative link property (arc balance) is proposed in 
[Shi \& Johansson, 2013] and strong connectedness is relaxed to quasi-strong connectedness. Finally, combinations of linear/nonlinear agent dynamic and linear/non linear (state dependent and/or time varying) interactions are considered in different way in the literature. In this direction agreement conditions for a class of both nonlinear time invariant and swithing/time varying networks are outlined in [Lin et al., 2007], [Hendrickx \& Tsitsiklis, 2013], [Slotine \& Wang, 2004], [Cao \& Ren, 2011], [Chopra \& Spong, 2007]. Recently in [Altafini, 2013] it is shown that bipartite consensus (i.e., the agents agree to a common absolute value) is achieved if and only if the signed graph of the network is structurally balanced.

\section{Paper Contribution}

In this paper we deal with agreement problem under minimal connectivity assumptions for networks of nonlinear time varying cooperative agents. It is still a challenging problem from both a theoretical and practical point of view to find easily checkable condition to assess agreement of nonlinear time-varying networks. This is due to the fact that the notion of connectivity becomes both state-dependent and time-dependent. The interplay of these two dimensions typically lends itself to connectivity conditions that need to be tested along solutions of the system.

In this respect our contributions are the following. Firstly, most of the network models considered in the literature of agreement problems are linear timevarying (or embedded in such models through the use of state-dependent weights) or nonlinear time invariant (i.e. [Hendrickx \& Tsitsiklis, 2013], [Altafini, 2013], [Lin et al., 2007]). Only few other approaches (i.e. [Slotine \& Wang, 2004]), to the best of our knowledge, explicitly consider nonlinear time varying networks. The latter, however, under instantaneous coupling conditions. Hereby we deal with nonlinear time varying monotone networks composed of monotone dynamical systems according the definition introduced in [Smith, 1995], therefore encompassing most of the agents models normally adopted in first-order consensus. Moreover, both the dynamic at the node (selffeedback) and the coupling can be time varying and state dependent (as it often occurs for instance in sensor robot networks).

Secondly, we propose a way of avoiding the circular argument by which solutions depend on the connectivity and the latter is in turn influenced by state evolutions. This type of circular argument normally makes up for conditions that can hardly be tested in the case of time-varying nonlinear agent dynamics and coupling without explicit apriori knowledge of solutions. Herein we propose a novel assumption on agent interactions and graph connectivity (later called: State-Frozen Integral Connectivity). In particular, our condition extends the use of integral connectivity-type condition of Moreau [Moreau, 2004] to nonlinear time varying networks by asking that a spanning tree exists for a suitably defined averaged graph. This only entails integrals performed in time on frozen state variables. Therefore, it enlarges the class of networks for which consensuability may be a priori and easier assessed with respect to related conditions in the literature that have to hold instantaneously ([Slotine \& Wang, 2004], [Hendrickx \& Tsitsiklis, 2013], [Martin \& Girard, 2013]) or require stronger graph connectivity ([Altafini, 2013]) or are restricted to specific class of nonlinear time invariant systems (i.e. [Cao \& Ren, 2011]). Finally, for the described class of systems, we relate State-Frozen connectivity to a seemingly weaker notion (later called Equilibrium Integral Connectivity) which may be tested by considering consensus states alone, and provide an estimate of the exponential rate of convergence towards the agreement space.

\section{$3 \quad$ Notation and problem statement}

Throughout the paper all vectors are assumed to be column vectors. To denote vectors we write $x=$ $\left(x_{1}, \ldots, x_{n}\right)$ for the column vector $x \in \mathbb{R}^{n} .|x|$ denotes the Euclidean norm of $x$. Let a compact set $\mathcal{K} \in \mathbb{R}^{n}$, herein we denote $\operatorname{diam}(\mathcal{K})=\sup \{|x-y|: x, y \in \mathcal{K}\}$. $\mathbf{1}$ is the vector of all ones and $e_{j}$ is the $j$-th element of the canonical basis of $\mathbb{R}^{n}$, where $n$ should normally be clear from the context. Given a function $f(t, x): \mathbb{R} \times \mathbb{R}^{n} \rightarrow \mathbb{R}^{n}$, piecewise continuous in $t$ and locally Lipschitz continuous with respect to $x$, the associated system of differential equations $\dot{x}(t)=f(t, x(t))$, is named cooperative if for any $i \in\{1,2, \ldots, n\}, f_{i}(t, x)$ is non-decreasing with respect to $x_{j}$ for all $j \neq i$. Notice that this condition implies monotonicity of the flow $\phi\left(t, x_{0}\right)$ with respect to initial conditions, namely, for all $t \geq 0$, it holds $\phi\left(t, x_{1}\right) \geq \phi\left(t, x_{2}\right)$ if $x_{1} \geq x_{2}$ (where " $\geq$ " is meant componentwise), [Smith, 1995].

Let $G(N, E)$ be a weighted directed graph (digraph) with the set of nodes $N=\{1, \ldots, n\}$, the set of edges $E \subseteq N \times N$. A node $j$ is reachable from node $i$ if there exists a path in a directed graph connecting nodes $i$ and $j$, namely there is a finite sequence $n_{1}, n_{2}, \ldots, n_{k}$ of distinct nodes such that $\left(n_{i}, n_{i+1}\right) \in E$ for $i=1, \ldots, k-1$ with $n_{1}=i$ and $n_{k}=j$. A digraph $G(N, E)$ is quasistrongly connected if there exists a node (root or center) from which any other node is reachable. $G(N, E)$ has a spanning tree if there exists a spanning tree that is a subgraph of $G$. Notice that the condition that $G(N, E)$ has a spanning tree is equivalent to quasi-strongly connectedness. A directed graph is connected if any two nodes can be joined by a path. A graph $G$ is called undirected if whenever $\left(n_{i}, n_{j}\right) \in E,\left(n_{j}, n_{i}\right) \in E$ as well. A network is described by a nonlinear dynamical system:

$$
\dot{x}(t)=f(t, x(t))
$$


where $x(t) \in \mathbb{R}^{n}$ is the state, $t \in \mathbb{R}_{+}$is the time variable and $f$ is the vector field $\mathbb{R}_{+} \times \mathbb{R}^{n} \rightarrow \mathbb{R}^{n}$. We denote with $F(t, x)=\left[F_{i j}(t, x)\right]$ the Jacobian matrix, when this can be defined.

We assume: $f$ is locally Lipschitz continuous with respect to $x$ uniformly in time, viz. for all compacts $K \in \mathbb{R}^{n}$ there exists $L_{K}>0$, such that, for all $x_{a}, x_{b} \in K$ and all $t \geq 0$ it holds $\left|f\left(t, x_{a}\right)-f\left(t, x_{b}\right)\right| \leq L_{K}\left|x_{a}-x_{b}\right|{ }^{1}$

The assumptions on $f$, imply the local existence and the unicity of the system's solution on some maximally extended open interval of definition. Fixed an arbitrary vector $x$, we define the following quantities: $x_{M}=\max _{k \in N}\left\{x_{k}\right\} ; x_{m}=\min _{k \in N}\left\{x_{k}\right\}$.

\section{Main Results}

We are now ready to state our main assumptions, which will guarantee exponential convergence towards a consensus state.

Assumption 1 We assume a cooperative (in the sense stated above) nonlinear network (1) with $f$ that admits an agreement equilibrium set, that is:

$$
\mathcal{E}=\operatorname{span}\{1\} \subseteq\left\{x \in \mathbb{R}^{n}: f(t, x)=0 \forall t \in \mathbb{R}_{+}\right\} .
$$

Definition 2 (Equilibrium interaction graph) We say that $G(N, E)$ is an equilibrium interaction graph for (1) if for all compact $\mathcal{K} \subseteq \mathbb{R}$, there exist $\varepsilon_{\mathcal{K}}>0$ and sufficiently large $T_{\mathcal{K}}>0$ so that for any $t \geq 0, \forall x \in \mathcal{K}$, $\forall(j, i) \in E$ it holds:

$$
\int_{t}^{t+T_{\mathcal{K}}} F_{i j}(\tau, x \mathbf{1}) d \tau \geq \varepsilon_{\mathcal{K}}
$$

\section{Definition 3 (State-Frozen interaction graph)}

We say that $G(N, E)$ is an interaction graph for (1) if for all compact $\mathcal{K} \subseteq \mathbb{R}$, there exist $\varepsilon_{\mathcal{K}}>0$ and sufficiently large $T_{\mathcal{K}}>0$ so that for any $t \geq 0, \forall x_{i}, x_{j} \in \mathcal{K}$, $\forall(j, i) \in E$ it holds:

$\operatorname{sign}\left(x_{j}-x_{i}\right) \int_{t}^{t+T_{\mathcal{K}}} f_{i}\left(\tau, x_{i} \mathbf{1}+e_{j}\left(x_{j}-x_{i}\right)\right) d \tau \geq \varepsilon_{\mathcal{K}}\left|x_{j}-x_{i}\right|$.

\section{Assumption 4 (Equilibrium semiglobal Integral Connectivity) \\ We say that network (1) fulfills semiglobal Integral Connec- tivity at equilibrium if it admits a quasi-strongly connected Equilibrium interaction graph. We denote by $\mathcal{T}_{r} \subseteq E$ and $r \in N$ the associated spanning tree and root node.}

\section{Assumption 5 (State-Frozen semiglobal Integral Connectivity)}

$\overline{1}$ This holds, for instance, when the Jacobian is uniformly bounded as a function of time.
We say that the network (1) fulfills State-Frozen semiglobal Integral Connectivity if it admits a quasi-strongly connected State-Frozen interaction graph. We denote by $\mathcal{T}_{r} \subseteq E$ and $r \in N$ the associated spanning tree and root node.

Assumption 4 along with local differentiability of $f(t, x)$ with respect to $x$ in a neighborhood of the equilibrium set $\mathcal{E}$ implies that around any equilibrium, there is an open region in which the system is contracting on average. Thanks to the monotonicity assumption, this property can be extended to the whole space as shown in the following Lemma 6.

Lemma 6 Given the complex network (1) and assuming that $f(t, x)$ is differentiable with respect to $x$ in a neighborhood of the equilibrium set with (uniformly in time) continuous derivatives then $G(N, E)$ is a State-Frozen interaction graph iff it is an Equilibrium interaction graph.

Proof Let $\mathcal{K} \subset \mathbb{R}$ be an arbitrary compact interval and $(j, i) \in E$ be an arbitrary edge in the Equilibrium interaction graph. We want to show that it is an edge also in the State-Frozen interaction graph. Let without loss of generality consider the case $x_{j}>x_{i}$, so that $\operatorname{sign}\left(x_{j}-x_{i}\right)=1$. By virtue of monotonicity, for any $\tilde{x}_{j}$ with $x_{i} \leq \tilde{x}_{j} \leq x_{j}$ we have:

$$
f_{i}\left(t, x_{i} \mathbf{1}+e_{j}\left(x_{j}-x_{i}\right)\right) \geq f_{i}\left(t, x_{i} \mathbf{1}+e_{j}\left(\tilde{x}_{j}-x_{i}\right)\right) .
$$

Let $\tilde{x}_{j}$ be close enough to $x_{i}$, so as to ensure continuous differentiability of $f$ with respect to $x$ (uniform in time), on the segment $x_{i} \mathbf{1}+s e_{j}\left(\tilde{x}_{j}-x_{i}\right), s \in[0,1]$. By the fundamental theorem of calculus, the following holds:

$$
\begin{array}{r}
f_{i}\left(\tau, x_{i} \mathbf{1}+e_{j}\left(\tilde{x}_{j}-x_{i}\right)\right)=f_{i}\left(\tau, x_{i} \mathbf{1}+e_{j}\left(\tilde{x}_{j}-x_{i}\right)\right)-f_{i}\left(\tau, x_{i} \mathbf{1}\right) \\
=\int_{0}^{1} F_{i j}\left(\tau, x_{i} \mathbf{1}+s e_{j}\left(\tilde{x}_{j}-x_{i}\right) s\right) d s\left(\tilde{x}_{j}-x_{i}\right) .
\end{array}
$$

Hence, taking averages over finite intervals of the above inequality and exchanging the order of integration yields:

$$
\begin{aligned}
& \int_{t}^{t+T} f_{i}\left(\tau, x_{i} \mathbf{1}+e_{j}\left(\tilde{x}_{j}-x_{i}\right)\right) d \tau \\
& =\int_{t}^{t+T}\left[f_{i}\left(\tau, x_{i} \mathbf{1}+e_{j}\left(\tilde{x}_{j}-x_{i}\right)\right)-f_{i}\left(\tau, x_{i} \mathbf{1}\right)\right] d \tau \\
& =\int_{0}^{1}\left(\int_{t}^{t+T} F_{i j}\left(\tau, x_{i} \mathbf{1}+s e_{j}\left(\tilde{x}_{j}-x_{i}\right)\right) d \tau\right) d s \cdot\left(\tilde{x}_{j}-x_{i}\right) .
\end{aligned}
$$

Notice that, by uniform continuity of derivatives there exists a class $\mathcal{K}$ function $\gamma_{\mathcal{K}}$ such that for all $(i, j)$ :

$$
F_{i j}\left(\tau, x_{i} \mathbf{1}+e_{j}\left(\tilde{x}_{j}-x_{i}\right) s\right) \geq F_{i j}\left(\tau, x_{i} \mathbf{1}\right)-\gamma_{\mathcal{K}}(s) .
$$

Hence, defining $\delta_{\mathcal{K}}:=\min \left\{1, \gamma_{\mathcal{K}}^{-1}\left(\varepsilon_{\mathcal{K}} / 2 T\right)\right\}$ yields $T \gamma_{\mathcal{K}}\left(\delta_{\mathcal{K}}\right) \leq \varepsilon_{\mathcal{K}} / 2$, and this can be exploited to derive 
the following bound:

$$
\begin{gathered}
\int_{0}^{1}\left(\int_{t}^{t+T} F_{i j}\left(\tau, x_{i} \mathbf{1}+e_{j}\left(\tilde{x}_{j}-x_{i}\right) s\right) d \tau\right) d s \\
\geq \int_{0}^{\delta_{\mathcal{K}}}\left(\int_{t}^{t+T} F_{i j}\left(\tau, x_{i} \mathbf{1}+e_{j}\left(\tilde{x}_{j}-x_{i}\right) s\right) d \tau\right) d s \\
\geq \int_{0}^{\delta_{\mathcal{K}}}\left(\int_{t}^{t+T} F_{i j}\left(\tau, x_{i} \mathbf{1}\right)-\gamma_{\mathcal{K}}(s) d \tau\right) d s \\
\geq \int_{0}^{\delta_{\mathcal{K}}}\left[\varepsilon_{\mathcal{K}}-T \gamma_{\mathcal{K}}(s)\right] d s \\
\geq \delta_{\mathcal{K}} \varepsilon_{\mathcal{K}}-T \gamma_{\mathcal{K}}\left(\delta_{\mathcal{K}}\right) \delta_{\mathcal{K}} \geq \delta_{\mathcal{K}} \varepsilon_{\mathcal{K}} / 2:=\tilde{\varepsilon}_{\mathcal{K}} .
\end{gathered}
$$

Combining the previous inequalities yields:

$$
\begin{gathered}
\int_{t}^{t+T} f_{i}\left(\tau, x_{i} \mathbf{1}+e_{j}\left(x_{j}-x_{i}\right)\right) d \tau \geq \\
\int_{t}^{t+T} f_{i}\left(\tau, x_{i} \mathbf{1}+e_{j}\left(\tilde{x}_{j}-x_{i}\right)\right) d \tau \geq \tilde{\varepsilon}_{\mathcal{K}},
\end{gathered}
$$

which proves that $(j, i)$ is an edge for the State-Frozen interaction graph provided we define $\tilde{\varepsilon}_{\mathcal{K}}$ as in (4) (notice that this definition is indeed independent of $i$ and $j$ ). Conversely if (3) holds then,

$$
\begin{gathered}
\int_{t}^{t+T} F_{i j}\left(\tau, x_{i} \mathbf{1}\right) d \tau= \\
\int_{t}^{t+T} \lim _{h \rightarrow 0} \frac{f_{i}\left(\tau, x_{i} \mathbf{1}+h e_{j}\right)-f_{i}\left(\tau, x_{i} \mathbf{1}\right)}{h} d \tau \\
=\lim _{h \rightarrow 0} \frac{\int_{t}^{t+T} f_{i}\left(\tau, x_{i} \mathbf{1}+h e_{j}\right) d \tau}{h} \geq \lim _{h \rightarrow 0} \frac{\varepsilon_{\mathcal{K}} h}{h}=\varepsilon_{\mathcal{K}} .
\end{gathered}
$$

Remark 7 Notice that if Assumptions 4-5 hold for some $T_{\mathcal{K}}$, it holds a fortiori for all $\tilde{T}_{\mathcal{K}}>T_{\mathcal{K}}$ (for the same compact $\mathcal{K}$ and same $\left.\varepsilon_{\mathcal{K}}\right)$. Informally speaking an edge in the graph from node $j$ to $i$ means that agent $j$ influences agent $i$ over the considered time horizon. Hence, existence of a spanning tree guarantees direct or indirect influence of the root node towards all other nodes (provided sufficient time is allowed). Notice that, in the light of Lemma 6, Assumptions 4 and 5 are equivalent. Condition (3) is an assumption of averaged quasi-strongly connectedness across uniform time intervals: despite its averaged character (which would more naturally lead to a test along solutions of (1)), the property is defined on frozen state variables, making easier its a priori verification. On the other side condition (2) is of really simple verification as it involves to test the condition just on the equilibrium solution. Notice that Definition 2 only needs the Jacobian to be defined locally around equilibrium states and (accordingly) the subsequent Lemma 6 assumes local differentiability of $f$ (uniform in time).

Let $x(t)$ denote an arbitrary solution of (1). The following fact is well-known and we prove it below only for the sake of completeness:

Lemma 8 The functions $x_{M}(t)$ and $x_{m}(t)$ are (respectively) monotonically non-increasing and nondecreasing.

Proof Equivalently we show that the set: $\mathcal{M}_{c}:=\{x$ : $\left.\max _{i \in N} x_{i} \leq c\right\}$, is forward invariant for all $c \in \mathbb{R}$. Let $x$ in $\mathcal{M}_{c}$ be arbitrary. Since $\mathcal{M}_{c}$ is convex, its tangent cone at $x$ is simply given by $T C_{x} \mathcal{M}_{c}=\left\{z: z_{i} \leq 0, \forall i: x_{i}=\right.$ $c\}$ (see Proposition 5.5, [Clarke et al., 1998]). Moreover, for all $i$ such that $x_{i}=c$ and any $t$ it holds, by cooperativity: $f_{i}(t, x) \leq f_{i}(t, c \mathbf{1})=0$.

Hence $f(t, x) \in T C_{x} \mathcal{M}_{c}$. As this holds for all $x \in \mathcal{M}_{c}$ it proves forward invariance of $\mathcal{M}_{c}$ (by Nagumo's Theorem - [Blanchini \& Miani, 2008]) and monotonicity of $x_{M}(t)$. A symmetric argument can be used to prove monotonicity of $x_{m}(t)$ by proving forward invariance of $\mathcal{N}_{c}=\left\{x: \min _{i \in N} x_{i} \geq 0\right\}$.

In what follows we will present the main lemmas which will allow us later to prove exponential consensus. We will consider as connectivity assumption the 5 (i.e. StateFrozen Integral Connectivity), remarking that the results we are presenting hold as well under assumption 4 by Lemma 6 . For sake of presentation we will omit the explicit depednence of $L, \varepsilon, T \mu$ from $\mathcal{K}$, with $\mathcal{K}$ being a compact set containing all agents at time instant $t=0$.

Lemma 9 Let $r \in N$ be the root of the spanning tree as from Assumption 5. For any $x \in \mathbb{R}^{n}$ let $\bar{x}$ and $\underline{x}$ denote the following:

$$
\begin{aligned}
& \bar{x}=x_{M} \mathbf{1}+\left(x_{r}-x_{M}\right) e_{r}, \\
& \underline{x}=x_{m} \mathbf{1}+\left(x_{r}-x_{m}\right) e_{r} .
\end{aligned}
$$

Let $\tilde{x}(\cdot)$ and $\underset{\sim}{x}(\cdot)$ denote the solutions of equation (1) from initial state $\bar{x}(t)$ at time $t($ viz. $\tilde{x}(\cdot)=\phi(\cdot-t, \bar{x}(t)))$ and from initial state $\underline{x}(t)$, respectively $($ viz. $\underset{\sim}{x}(\cdot)=\phi(\cdot-$ $t, \underline{x}(t))$. If there exist a finite positive integer $\tilde{\bar{k}}$ and $\mu>0$ (uniform in $t$ ) such that:

$$
\tilde{x}_{M}(t+\bar{k} T) \leq x_{M}(t)-\mu\left|x_{M}(t)-\tilde{x}_{r}(t)\right|
$$

and respectively:

$$
{\underset{\sim}{x}}_{m}(t+\bar{k} T) \geq x_{m}(t)+\mu\left|x_{m}(t)-{\underset{\sim}{x}}_{r}(t)\right|,
$$

then, similar inequalities hold for the solution $x(\cdot)$, viz.:

$$
x_{M}(t+\bar{k} T) \leq x_{M}(t)-\mu\left|x_{M}(t)-x_{r}(t)\right|
$$


and:

$$
x_{m}(t+\bar{k} T) \geq x_{m}(t)+\mu\left|x_{m}(t)-x_{r}(t)\right| .
$$

Proof Notice that, by construction $\bar{x}(t) \geq x(t)$. Hence, by monotonicity, $\tilde{x}(\tau) \geq x(\tau)$ holds componentwise for all $\tau \geq t$ and implies $\tilde{x}_{M}(\tau) \geq x_{M}(\tau)$. In particular, since $\tilde{x}_{M}(t)=x_{M}(t)$ and $\tilde{x}_{r}(t)=x_{r}(t)$, it is enough to prove inequality (5) to guarantee inequality (6). A symmetric argument applies to $\underset{\sim}{x}(\cdot)$.

Informally speaking the previous Lemma states that there exists a worst-case scenario for the initial distribution of agents when it comes to the rate of convergence towards consensus. This is described by the initial condition $\bar{x}$ (or $\underline{x}$, respectively): a condition in which all agents have maximum (respectively minimum) value, except for the root node $r$.

Lemma 10 Let $r \in N$ be the root of the spanning tree as from Assumption 5. There exists a finite positive integer $\bar{k}$ and $\mu>0$ (uniform in $t$ ) such that (6) holds.

Proof Let $d(k): N \rightarrow \mathbb{N}$ denote the distance in the spanning tree of node $k$ from the root of the tree in Assumption 5, which we denote by $r$. For nodes $k$ with $d(k)=1$ and all $\tau \in[t, t+2 T]$ the following holds:

$$
\begin{aligned}
& \tilde{x}_{k}(\tau)-x_{M}(t)=\int_{t}^{\tau} f_{k}(\theta, \tilde{x}(\theta)) d \theta \\
& \leq \int_{t}^{\tau} f_{k}\left(\theta, x_{M}(t) \mathbf{1}+\left(\tilde{x}_{r}(\theta)-x_{M}(t)\right) e_{r}\right. \\
& \left.+\left(\tilde{x}_{k}(\theta)-x_{M}(t)\right) e_{k}\right) d \theta
\end{aligned}
$$

where the inequality holds from non-decreasingness of $f_{k}$ with respect to all $x_{i} \mathrm{~s}$, with $i \neq k$, and remarking that for all $\theta \geq t, \tilde{x}(\theta) \leq \tilde{x}_{M}(\theta) \mathbf{1} \leq \tilde{x}_{M}(t) \mathbf{1}=x_{M}(t) \mathbf{1}$. This last inequality is instrumental for the subsequent exploitation of Lipschitz continuity of $f$. Indeed, we may observe that:

$$
\begin{aligned}
& \int_{t}^{\tau}\left[f _ { k } \left(\theta, x_{M}(t) \mathbf{1}+\left(\tilde{x}_{r}(\theta)-x_{M}(t)\right) e_{r}\right.\right. \\
& \left.+\left(\tilde{x}_{k}(\theta)-x_{M}(t)\right) e_{k}\right) \\
& \left.-f_{k}\left(\theta, x_{M}(t) \mathbf{1}+\left(\tilde{x}_{r}(\theta)-x_{M}(t)\right) e_{r}\right)\right] d \theta \\
& \leq-L \int_{t}^{\tau}\left[\tilde{x}_{k}(\theta)-x_{M}(t)\right] d \theta,
\end{aligned}
$$

where $L$ is the time independent Lipschitz constant of $f_{k}$. Combining inequalities (7) and (8) we see that:

$$
\begin{aligned}
& \tilde{x}_{k}(\tau)-x_{M}(t) \leq \\
& \int_{t}^{\tau} f_{k}\left(\theta, x_{M}(t) \mathbf{1}+\left(\tilde{x}_{r}(\theta)-x_{M}(t)\right) e_{r}\right) d \theta
\end{aligned}
$$

$$
\begin{aligned}
& -L \int_{t}^{\tau}\left[\tilde{x}_{k}(\theta)-x_{M}(t)\right] d \theta \\
& \leq \int_{t}^{\tau} f_{k}\left(\theta, x_{M}(t) \mathbf{1}+\left(\hat{x}_{r}-x_{M}(t)\right) e_{r}\right) d \theta \\
& -L \int_{t}^{\tau}\left[\tilde{x}_{k}(\theta)-x_{M}(t)\right] d \theta
\end{aligned}
$$

where the last inequality is derived considering that $\hat{x}_{r}=$ $\max _{\theta \in[t, t+2 T]} \tilde{x}_{r}(\theta)$. In particular, thanks to condition (3) and Assumption 5 applied to the first term of the latter inequality for $\tau \in[t+T, t+2 T],(j, i)=(r, k)$, $x_{i}=x_{M}(t)$ and $x_{j}=\hat{x}_{r}$ we see that:

$$
\begin{aligned}
& \tilde{x}_{k}(\tau)-x_{M}(t) \leq-\varepsilon_{\mathcal{K}}\left(x_{M}(t)-\hat{x}_{r}\right) \\
& -L \int_{t}^{\tau}\left[\tilde{x}_{k}(\theta)-x_{M}(t)\right] d \theta ;
\end{aligned}
$$

(notice in this respect that the subscripts $i$ and $j$, of $x_{i}$, $x_{j}$ in Assumption 5 are merely typographical symbols, and bear no connection with the edge $(j, i)$ under consideration). By defining $\Delta(\tau)=\int_{t}^{\tau}\left[\tilde{x}_{k}(\theta)-x_{M}(t)\right] d \theta$ we can recast equation (9) as:

$$
\frac{d}{d \tau} \Delta(\tau) \leq-\varepsilon_{\mathcal{K}}\left(x_{M}(t)-\hat{x}_{r}\right)-L \Delta(\tau),
$$

which holds for all $\tau \in[t+T, t+2 T]$. Since $\Delta(t+T) \leq 0$, by a standard comparison principle we see that:

$$
\Delta(\tau) \leq-\frac{\varepsilon_{\mathcal{K}}}{L}\left(x_{M}(t)-\hat{x}_{r}\right)\left[1-e^{-L(\tau-T-t)}\right],
$$

which holds for all $\tau \in[t+T, t+2 T]$. In particular, for $\tau=t+2 T$ equation (10) yields:

$$
\Delta(t+2 T) \leq-\frac{\varepsilon_{\mathcal{K}}}{L}\left(x_{M}(t)-\hat{x}_{r}\right)\left[1-e^{-L T}\right]
$$

From the mean value theorem it results:

$$
\exists t^{*} \in[t, t+2 T]: \tilde{x}_{k}\left(t^{*}\right)-x_{M}(t)=\frac{\Delta(t+2 T)}{2 T} .
$$

By Lipschitz continuity of $f$, convergence of $\tilde{x}_{k}(t)$ to $x_{M}(t)$ is at most exponential (within time $t+2 T$ ), and therefore we may infer:

$$
\begin{aligned}
& \tilde{x}_{k}(t+2 T)-x_{M}(t) \leq\left(\tilde{x}_{k}\left(t^{*}\right)-x_{M}(t)\right) e^{-L\left(t+2 T-t^{*}\right)} \\
& \leq\left(\tilde{x}_{k}\left(t^{*}\right)-x_{M}(t)\right) e^{-2 L T}
\end{aligned}
$$

From (12) and (13) it results:

$$
\tilde{x}_{k}(t+2 T)-x_{M}(t) \leq \frac{\Delta(t+2 T)}{2 T} e^{-2 L T}
$$


Finally, in order to derive an estimate of how decreasing is $\tilde{x}_{k}(t)$ which is uniform in time we need to observe:

$$
\begin{aligned}
& \left|\tilde{x}_{r}(\theta)-x_{M}(t)\right| \geq\left|\tilde{x}_{r}(\theta)-x_{M}(\theta)\right| \\
& \geq e^{-2 L T}\left|\tilde{x}_{r}(t)-x_{M}(t)\right|, \quad \forall \theta \in[t, \tau] .
\end{aligned}
$$

that along with the folllowing:

$$
\left|\hat{x}_{r}-x_{M}(t)\right|=\min _{\theta \in[t, \tau]}\left|\tilde{x}_{r}(\theta)-x_{M}(t)\right|,
$$

yields to:

$$
\left|\hat{x}_{r}-x_{M}(t)\right| \geq e^{-2 L T}\left|\tilde{x}_{r}(t)-x_{M}(t)\right| .
$$

In fact, by combining (11), (14) and (15) we obtain:

$\tilde{x}_{k}(t+2 T)-x_{M}(t) \leq-\varepsilon_{\mathcal{K}} \frac{e^{-4 L T}}{2 L T}\left[1-e^{-L T}\right]\left|\tilde{x}_{r}(t)-x_{M}(t)\right|$.

Next we deal with nodes $k \in N$ with $d(k)=2$. Let $j$ be such that $d(j)=1$ and $(j, k) \in E$. The following holds for all $\tau \in[t+2 T, t+4 T]$ :

$$
\begin{aligned}
& \tilde{x}_{k}(\tau)-\tilde{x}_{k}(t+2 T)=\int_{t+2 T}^{\tau} f_{k}(\theta, \tilde{x}(\theta)) d \theta \\
& \leq \int_{t+2 T}^{\tau} f_{k}\left(\theta, x_{M}(t) \mathbf{1}+\left(\tilde{x}_{j}(\theta)-x_{M}(t)\right) e_{j}\right. \\
& \left.+\left(\tilde{x}_{k}(\theta)-x_{M}(t)\right) e_{k}\right) d \theta \\
& \leq \int_{t+2 T}^{\tau} f_{k}\left(\theta, x_{M}(t) \mathbf{1}+\left(\tilde{x}_{j}(\theta)-x_{M}(t)\right) e_{j}\right) d \theta \\
& -L \int_{t+2 T}^{\tau}\left[\tilde{x}_{k}(\theta)-x_{M}(t)\right] d \theta \\
& \leq \int_{t+2 T}^{\tau} f_{k}\left(\theta, x_{M}(t) \mathbf{1}+\left(\hat{x}_{j}-x_{M}(t)\right) e_{j}\right) d \theta \\
& -L \int_{t+2 T}^{\tau}\left[\tilde{x}_{k}(\theta)-x_{M}(t)\right] d \theta
\end{aligned}
$$

where $\hat{x}_{j}=\max _{\theta \in[t+2 T, t+4 T]} \tilde{x}_{j}(\theta)$. In particular then, for all $\tau \in[t+3 T, t+4 T]$ we see that:

$$
\begin{aligned}
& \tilde{x}_{k}(\tau)-x_{M}(t) \leq \tilde{x}_{k}(\tau)-\tilde{x}_{k}(t+2 T) \\
& \leq-\varepsilon_{\mathcal{K}}\left(x_{M}(t)-\hat{x}_{j}\right)-L \int_{t+2 T}^{\tau}\left[\tilde{x}_{k}(\theta)-x_{M}(t)\right] d \theta
\end{aligned}
$$

By defining $\Delta(\tau)=\int_{t+2 T}^{\tau}\left[\tilde{x}_{k}(\theta)-x_{M}(t)\right] d \theta$ we can recast equation (17) as:

$$
\frac{d}{d \tau} \Delta(\tau) \leq-\varepsilon_{\mathcal{K}}\left(x_{M}(t)-\hat{x}_{j}\right)-L \Delta(\tau)
$$

which holds for all $\tau \in[t+3 T, t+4 T]$. Since $\Delta(t+3 T) \leq$
0, by a standard comparison principle we see that:

$$
\Delta(\tau) \leq-\frac{\varepsilon_{\mathcal{K}}}{L}\left(x_{M}(t)-\hat{x}_{j}\right)\left[1-e^{-L(\tau-3 T-t)}\right],
$$

which holds for all $\tau \in[t+3 T, t+4 T]$. In particular, for $\tau=t+4 T$ equation (18) yields:

$$
\Delta(t+4 T) \leq-\frac{\varepsilon_{\mathcal{K}}}{L}\left(x_{M}(t)-\hat{x}_{j}\right)\left[1-e^{-L T}\right] .
$$

By applying the mean value theorem and exploiting Lipschitz continuity, we get:

$$
\tilde{x}_{k}(t+4 T)-x_{M}(t) \leq \frac{\Delta(t+4 T)}{2 T} e^{-2 L T}
$$

Consequently, in order to derive an estimate of how decreasing is $\tilde{x}_{k}(t)$ which is uniform in time we need to remark that by uniform Lipschitz continuity and exploiting (16) applied to node $j$ at distance $d(j)=1$ from $r$ we get:

$$
\begin{aligned}
& \left|\hat{x}_{j}-x_{M}(t)\right| \geq e^{-2 L T}\left|\tilde{x}_{j}(t+2 T)-x_{M}(t)\right| \\
& \geq \varepsilon_{\mathcal{K}} \frac{e^{-6 L T}}{2 L T}\left[1-e^{-L T}\right]\left|\tilde{x}_{r}(t)-x_{M}(t)\right| .
\end{aligned}
$$

Finally, by combining (19), (20) and (21) we obtain:

$$
\begin{aligned}
& \tilde{x}_{k}(t+4 T)-x_{M}(t) \\
& \leq-e^{-8 L T}\left(\frac{\varepsilon_{\mathcal{K}}\left[1-e^{-L T}\right]}{2 L T}\right)^{2}\left|\tilde{x}_{r}(t)-x_{M}(t)\right| .
\end{aligned}
$$

A similar procedure can be used to construct an estimate of the convergence rate for an arbitrary node at distance $d(k)+1$ based on the estimate for nodes at distance $d(k)$. By induction, for any node $k$ at distance $d(k)$ from the root, the following inequality holds:

$$
\begin{aligned}
& \tilde{x}_{k}(t+2 d(k) T)-x_{M}(t) \\
& \leq-e^{-(4 d(k) L T)}\left(\frac{\varepsilon_{\mathcal{K}}\left[1-e^{-L T}\right]}{2 L T}\right)^{d(k)}\left|\tilde{x}_{r}(t)-x_{M}(t)\right| \\
& =-\mu(d(k))\left|\tilde{x}_{r}(t)-x_{M}(t)\right|,
\end{aligned}
$$

with $\mu(d(k))=e^{-(4 d(k) L T)}\left(\frac{\varepsilon_{\mathcal{K}}\left[1-e^{-L T}\right]}{2 L T}\right)^{d(k)}$ being a positive constant for any fixed $d(k)$.

Given the fact that only a finite number of agents are present, considering monotonicity of $x_{M}(t)$ and, by Assumption 5 , every agent $k$ having a finite distance from the root, a uniform estimate of the convergence rate can be provided letting $\bar{k}:=2(n-1)$ and $\mu=\mu(\bar{k} / 2)=$ $\mu(n-1)$. This concludes the proof of inequality (5) that by Lemma 9 yields to inequality (6). 
Theorem 11 Consider the network modeled by equations (1), if Assumption 5 holds, then the equilibrium set $\mathcal{E}$ is uniformly exponentially stable and $x(t)$ converges exponentially to an agreement equilibrium state.

Proof We prove the result by a Lyapunov argument. Consider the function:

$$
V(x)=\max _{k \in N} x_{k}-\min _{k \in N} x_{k}
$$

This is positive definite and radially unbounded with respect to the equilibrium set $\mathcal{E}$. Moreover, by Lemma 8 it is non-increasing along solutions. From Lemma 9 and 10 more is actually true, in particular there exist a finite $\bar{k}$ and positive $\mu(\bar{k} / 2) \in(0,1)$ such that:

$$
V(x(t+\bar{k} T))-V(x(t)) \leq-\mu(\bar{k} / 2) V(x(t)) .
$$

Inequality (22) proves Exponential Uniform Asymptotic Stability of the equilibrium set and exponential convergence of $V(x)$ to 0 . Moreover, as $x_{M}(t)$ and $x_{m}(t)$ are monotone and bounded functions of time both of them are converging and their limits are equal. Therefore, $x(t)$ approaches asymptotically a single equilibrium that belongs to $\mathcal{E}$, viz. exponential asymptotic consensus is achieved.

\section{$5 \quad$ Representative Examples}

In this Section we will discuss illustrative examples showing the merits of the proposed conditions. Firstly we consider a network composed of 4 agents connected bidirectionally in a ring topology:

$$
\dot{x}_{i}=\sigma\left(t+x_{i}\right) \operatorname{sat}\left(x_{i+1}-x_{i}\right)+\sigma\left(t+x_{i}\right) \operatorname{sat}\left(x_{i-1}-x_{i}\right)
$$

for $i=1,2,3,4$ with the notation that $x_{0}=x_{4}$ and $x_{5}=$ $x_{1}$, while $\sigma(\cdot)$ is a periodic function represented in Fig. 1-(a) with period $T=2$ and sat denotes the standard symmetric piecewise-linear saturation. By verification of (2) in Assumption 4 for $i=1,2,3$, 4 , it results:

$$
\begin{aligned}
& \int_{t}^{t+T} F_{i i \pm 1}(\tau, x \mathbf{1}) d \tau=\int_{t}^{t+T} \sigma(\tau+x) d \tau \\
& =\int_{0}^{T} \sigma(\tau) d \tau \geq \varepsilon_{\mathcal{K}},
\end{aligned}
$$

provided $\varepsilon_{\mathcal{K}}:=\int_{0}^{T} \sigma(\tau) d \tau$. Similarly, condition (3) in Assumption 5 may be used to assess consensus: indeed its verification for $i=1,2,3,4$, yields to:

$$
\begin{gathered}
\operatorname{sign}\left(x_{i \pm 1}-x_{i}\right) \int_{t}^{t+T} \sigma\left(\tau+x_{i}\right) \operatorname{sat}\left(x_{i \pm 1}-x_{i}\right) d \tau= \\
\operatorname{sign}\left(x_{i \pm 1}-x_{i}\right) \operatorname{sat}\left(x_{i \pm 1}-x_{i}\right) \int_{t}^{t+T} \sigma\left(\tau+x_{i}\right) d \tau
\end{gathered}
$$

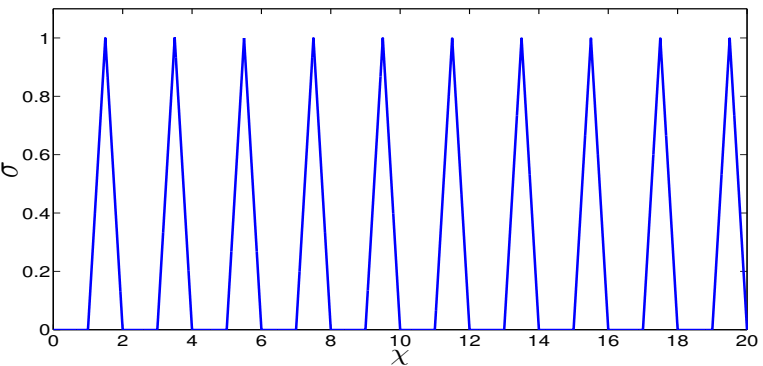

(a)

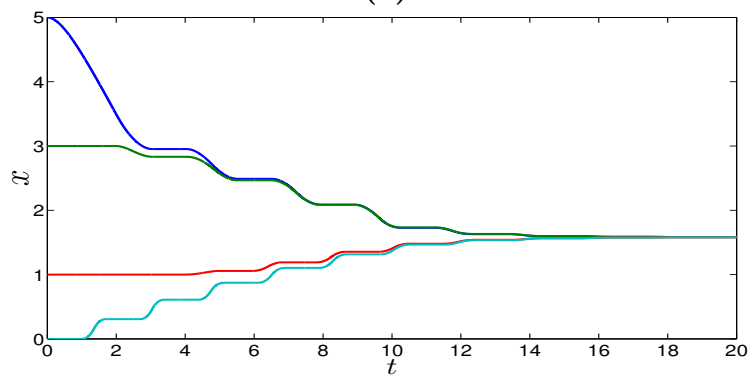

(b)

Fig. 1. Asymptotic convergence to the consensus state: (a) $\sigma(\chi)$ (b) dynamic evolution of $x$.

$$
=\left|\operatorname{sat}\left(x_{i \pm 1}-x_{i}\right)\right| \cdot \int_{0}^{T} \sigma(\tau) d \tau \geq \varepsilon_{\mathcal{K}}\left|x_{i \pm 1}-x_{i}\right| .
$$

provided $T_{\mathcal{K}}=T, \varepsilon_{\mathcal{K}}:=\min \{1,1 / \operatorname{diam}(\mathcal{K})\} \int_{0}^{T} \sigma(\tau) d \tau$. From (24) and (25) it appears that all the required Assumptions are fulfilled (any node can be taken to be the root given the ring-topology of the graph of interactions). It is worth pointing out that integration in (24) and (25) are performed with "frozen" state variables, greatly simplifying the a priori verification of the conditions guaranteeing asymptotic consensus, (see Fig. 1(b) for a simulation). Additionally the verification of the condition (2) is further simplified just considering the integration around the equilibrium space.

Now we consider a different class of examples for which consensus can be assessed by using our conditions. Let $G(N, E)$ be a quasi-strongly connected directed graph, with $N_{i}$ being the set of nodes $j$ such that $(j, i)$ belongs to $E$. We consider the following set of equations:

$$
\dot{x}_{i}=-2 x_{i}+\min _{j \in N_{i} \cup\{i\}}\left\{x_{j}\right\}+\max _{j \in N_{i} \cup\{i\}}\left\{x_{j}\right\} .
$$

More in general, one could allow for a time-varying graph $G(N, E(t))$, and introduce time-varying weights as follows:

$$
\begin{aligned}
& \dot{x}_{i}=-\alpha_{m}(t)\left(x_{i}-\min _{j \in N_{i}(t) \cup\{i\}} x_{j}\right) \\
& -\alpha_{M}(t)\left(x_{i}-\max _{j \in N_{i}(t) \cup\{i\}} x_{j}\right) .
\end{aligned}
$$


Verification of the proposed Assumption 5 on equation (26), for any $j \in N_{i}$ yields:

$$
\begin{aligned}
& \operatorname{sign}\left(x_{j}-x_{i}\right) \int_{t}^{t+T_{\mathcal{K}}}\left(-2 x_{i}+\min \left\{x_{j}, x_{i}\right\}+\max \left\{x_{j}, x_{i}\right\}\right) d \tau \\
& =\operatorname{sign}\left(x_{j}-x_{i}\right) \int_{t}^{t+T_{\mathcal{K}}}\left(-2 x_{i}+x_{i}+x_{j}\right) d \tau \geq \varepsilon_{\mathcal{K}}\left|x_{j}-x_{i}\right|
\end{aligned}
$$

holding for any $0<\varepsilon_{\mathcal{K}} \leq T_{\mathcal{K}}$. Notice that application of condition (2) is not straightforward due to nonsmoothness of the functions $f_{k}$. Therefore, assumption 4 , albeit of more direct verification, is less general than assumption 5 .

\section{Discussion}

We provide below a comparison of the new conditions introduced with respect to many interesting ones already available in the literature. The aim of this Section is to further remark how the proposed conditions are independent on the existing conditions, and how their verification may sometimes be simpler and more direct even if existing conditions could be also applied.

We start with an interesting assumption A2, pg.5 in [Lin et al., 2007] named strict sub-tangentiality condition. It represents the continous-time counterpart of Moreau's assumptions and it is used to prove asymptotic consensus for uniformly quasi-strongly connected interaction graphs for nonlinear time-invariant systems in the presence of switching (in time) topologies. Reference [Lin et al., 2007] also mentions the corresponding condition for time-varying vector fields but claims that this is not enough, in general, to guarantee asymptotic consensus. Decoupling state-dependence from time-dependence (through a switching topology) is one of the crucial technical ingredients that allow to guarantee consensus. Cooperativity of the dynamical system is, however, not required. In the case of cooperative nonlinear networks, with switching topology and one-dimensional agents' states, the above mentioned condition is very similar to the one proposed here (3), one slight difference being that condition A2 involves all neighbors configurations of agent $i$-th while (3) only considers one neighbor at a time being displaced from the consensus configuration. As a matter of fact, the proof in [Lin et al., 2007] borrows heavily Moreau's geometric concepts and proof's structure, pointing out remarkable challenges presented in the continous-time case, while the proof herein developed strongly relies on the flow monotonicity allowed by the considered scenario of cooperative networks. For this scenario, we further extend to the time-varying case the above strict sub-tangentiality-like condition while, by making strictness linear-like, we additionally guarantee exponential convergence.

Another remarkable condition dealing with a class of networked nonlinear systems coupled by a matrix $D(t)$ is proposed in [Qu, 2009]. Specifically, the notion of strict relative amplitude dominant network (i.e. condition 6.7) is introduced that, along with the condition of uniform sequential completeness of $D(t)$ ensures asymptotic consensus. The proposed notion of connectivity, however, requires a special kind of network's equation for which connectivity coefficients enter in a multiplicative way. This is not necessary the case in the our approach and examples (26) and (27) introduced above show that providing a time-varying linear-like embedding may result in connectivity conditions which are hard to test a priori. This will be highlighted later by introducing the embedding (28). Therefore, the conditions herein proposed may be simpler to test as specifically pointed out by examples (26) and (27). Finally, exponential convergence is hereby guaranteed.

Finally, the condition (2) has no obvious counterpart in [Lin et al., 2007] and [Qu, 2009], or in the existing literature on consensus for nonlinear networks.

Now we are recalling some notable connectivity conditions for first order time varying consensus protocols. We start with the well-known assumption of integral connectivity introduced by Moreau ([Moreau, 2004]) states that:

Assumption 12 (Integral-connectivity) The link weights $a_{i j}(t)$ are uniformly bounded and there exist $T>0$ and $\delta>0$ such that the directed graph obtained by connecting $i$ to $j$ whenever $\int_{t}^{t+T} a_{i j}(\tau) d \tau>\delta$ for every $t$, has a rooted spanning tree.

The presented Assumptions 4 and 5 enlarges the class of networks for which consensus may be assessed under "integral connectivity" type condition to nonlinear time varying monotone systems, with the remarkable feature of being frozen in state variables, that makes its direct verification more straightforward. In addition to [Moreau, 2004], we provide an estimate (albeit conservative) of the exponential rate of convergence towards the agreement space.

In [Hendrickx \& Tsitsiklis, 2013] the following cutbalanced assumption is presented:

Assumption 13 (Cut-balance) There exists a constant $K \geq 1$ such that for all $t$, and any nonempty subset $S$ of $\{1, \ldots, n\}$, it holds:

$$
K^{-1} \sum_{i \in S, j \notin S} a_{j i}(t) \leq \sum_{i \in S, j \notin S} a_{i j}(t) \leq K \sum_{i \in S, j \notin S} a_{j i}(t)
$$

In [Martin \& Girard, 2013] the authors extend the continuous time result in [Hendrickx \& Tsitsiklis, 2013] by replacing the Assumption 13 with the slow divergence of reciprocal weights. Let $r(t)=\max _{S \neq \emptyset, S \varsubsetneqq N} r_{S}(t)$, with $r_{S}(t)$ being the ratio between reciprocal interaction weights defined as: $\left[\sum_{i \in S, j \notin S} a_{i j}(t)\right] /\left[\sum_{i \in S, j \notin S} a_{j i}(t)\right]$ if the denominator is positive; 1 if the numerator and 
denominator are equal to zero; $+\infty$ if the denominator is zero and the numerator is positive. The Assumption introduced in [Martin \& Girard, 2013] states:

Assumption 14 (slow divergence of reciprocal weights) For all $t \geq 0, r(t)$ is finite and the infinite sum $\sum_{p \in \mathbb{N}} r\left(t_{p}\right)^{-\left[\frac{n}{2}\right]}=+\infty$

Assumption 14 enables the divergence of reciprocal interaction weights provided this divergence gets slower and slower as larger is the number of agents.

An alternative link property (arc balance) is proposed in [Shi \& Johansson, 2013] where the graph strong connectivity is relaxed to be quasi strongly connected:

Assumption 15 (arc balance) There exists a constant $K>1$ such that for any two arcs $(j, i),(m, k)$ and $t \geq 0$, it results: $K^{-1} a_{i j}(t) \leq a_{k m}(t) \leq K a_{i j}(t)$.

In [Shi \& Johansson, 2013] a relation between 15 and 13 is outlined. Assumption 13 guarantees cluster consensus rather consensus. The remaining assumptions, except 14 and 15 that also ensure exponential convergence rates, along with infinite integral connectivity-type assumptions (e.g. persistent connectivity, unbounded interactions connectivity) guarantee asymptotic convergence to consensus. Additionally they consider linear time varying first order networks (and/or state dependent systems embedded in such models through the use of statedependent weights [Hendrickx \& Tsitsiklis, 2013]). Finally all of them have to hold instantaneously even though this does not imply interaction graph that is strongly connected at all times. Differently, Assumptions 4 and 5 have simultaneously the features to require the existence of a spanning tree for a graph defined by means of a time average of interaction strength performed over all state values (frozen in time) or just around the equilibrium, and to be applied to nonlinear time varying monotone systems to assess exponential consensus.

In [Altafini, 2013] the notion of bipartite consensus is introduced for signed graph networks in which the edges can assume also negative weights. Remarkably, the relation between strong monotonicity property and bipartite consensus achievement is pointed out. Specifically, any strongly monotone system ([Smith, 1995]) whose Jacobian $F(x)$ is globally $\epsilon$-strongly connected (e.g. the graph obtained by connecting $i$ to $j$ whenever $|F(x)| \geq \epsilon$ is strongly connected) can be turned into a (nonlinear) Laplacian scheme achieving bipartite consensus. Herein, we assess consensus under quasi-strongly connectivity property (just the existence of a spanning tree for a suitable averaged graph is required) for a larger class of nonlinear time varying (not strongly) monotone networks.

We remark the different nature of Interaction Graphs proposed in Definition 2 and 3. The first one has to hold only on equilibrium states (equilibrium set $\mathcal{E}$ ) provided $f(t, x)$ is $C^{1}$ in a neighborhood of $\mathcal{E}$. The second one, Definition 3 , has to hold for edge $(j, i)$ on all state configurations in which all agents except $j$ take the same value of agent $i$. Interestingly, they are equivalent as shown by Lemma 6 . This says how the proposed condition are not stronger than similar conditions formulated along all solutions, usually considered for nonlinear systems. Hence, the proposed criteria are different and independent of existing conditions. Additionally, they may be of easier verification even for time-invariant network's scenarios. To highlight this feature let consider the representative example earlier introduced by system (26). A standard technique to apply the conditions holding for linear time-varying protocols is to regard (26) as embedded in the following linear time-varying system:

$$
\dot{x}_{i}=\sum_{j \neq i} a_{i j}(t)\left(x_{j}-x_{i}\right)
$$

where the coefficients $a_{i j}(t)$ are regarded as exogenous input computed in feedback according to:

$$
a_{i j}(t)=\left\{\begin{array}{c}
1 / \operatorname{card}\left(\operatorname{argmin}_{k \in N_{i}} x_{k}(t)\right), \\
\quad \text { if } j \in \operatorname{argmin}_{k \in N_{i}} x_{k}(t) ; \\
1 / \operatorname{card}\left(\operatorname{argmax}_{k \in N_{i}} x_{k}(t)\right), \\
\quad \text { if } j \in \operatorname{argmax}_{k \in N_{i}} x_{k}(t) ; \\
0, \quad \text { otherwise. }
\end{array}\right.
$$

Notice that, according to this embedding, each agent is (for almost all values of $t$ ) connected to at most two of its neighbours. Therefore, verification of connectivity conditions according to Moreau's definition is not straightforward and might fail, especially for graphs with a large number of neighbours at each node but a relatively low number of loops (for instance 0 loops as in the case of trees). Similar difficulties are encountered with all available criteria for consensus of time-varying linear networks, including the approach proposed in [Qu, 2009]. Indeed, in order to attack this example with embedding techniques, a more subtle embedding must be employed. The one we proposed is, however, the easiest and most natural one. Subtangentiality conditions as in [Lin et al., 2007] apply to system (26), and guarantee asymptotic consensus, even in the case of time-varying graphs $G(N, E(t))$, as this correspond to hypothesis of switching topology. The fully time-varying set-up, with time-varying weights as in equation $(27)$, is instead not covered by [Lin et al., 2007].

Finally the proposed approach can encompass existing agreement conditions also in the case of simple timeinvariant models. A trivial representative example is the following:

$$
\begin{aligned}
& \dot{x}_{1}(t)=-\operatorname{sat}\left(x_{1}-x_{2}\right) \\
& \dot{x}_{2}(t)=-\operatorname{sat}\left(x_{2}-x_{1}\right)
\end{aligned}
$$


for which "Jacobian-based" conditions are inconclusive given the apparent loss of connectivity experienced when operating in the saturated region over the threshold 1 , namely: $\frac{\partial \dot{x}_{1}}{\partial x_{2}}=\frac{\partial \dot{x}_{2}}{\partial x_{1}}=0, \forall\left|x_{1}-x_{2}\right| \geq 1$.

\section{Conclusions and Future work}

This paper introduces conditions for exponential agreement (state frozen integral connectivity and equilibrium integral connectivity), suitable for nonlinear cooperative time-varying networks. The conditions extend, to this nonlinear scenario, the notion of integral connectivity well known in the literature and have the additional merit to be frozen in state variables to allow for a priori simpler verification. Remarkably, the seemingly weaker notion of equilibrium integral connectivity, only involves Jacobian connectivity on equilibrium configurations for networks defined through sufficiently smooth equations. As a corollary, exponential convergence to the agreement space is proved and an estimate of the convergence rate is given. Ongoing work is devoted to assess agreement conditions for non-monotone networks.

\section{Acknowledgements}

The authors would like to thank the anonymous reviewers for their valuable comments and suggestions that helped improve the quality of the paper.

\section{References}

[Murray, 2007] R.M. Murray (2007), Recent Research in Cooperative Control of Multivehicle Systems Journal of Dynamic Systems, Measurement, and Control, 129, 5.

[Manfredi E Angeli, 2013] S. Manfredi, D. Angeli (2013) Frozen State Conditions for Asymptotic Consensus of Time-Varying Cooperative Nonlinear Networks 52nd IEEE Conference on Decision and Control, 1325 - 1330, December, Firenze, Italy.

[Blanchini 8 Miani, 2008] F. Blanchini and S. Miani (2008) Set Theoretic Methods in Control, Systems and Control Foundations and Applications, Birkhauser.

[Chopra ES Spong, 2007] N. Chopra, M. Spong, (2007) Passivity Based Control of Multi-Agent Systems. Advances in Robot Control From Everyday Physics in Human-Like Movements, 107-134.

[Moreau, 2005] L. Moreau (2005). Stability of multiagent systems with time-dependent communication links. IEEE Transactions on Automatic Control, 50, 2.

[Moreau, 2004] L. Moreau (2004). Stability of continuous-time distributed consensus algorithms, Proc. 43rd IEEE Conf. Decision and Control.

[Hendrickx 8 Tsitsiklis, 2013] J. M. Hendrickx and J. N. Tsitsiklis. (2013) Convergence of type-symmetric and cutbalanced consensus seeking systems. IEEE Transactions on Automatic Control, 58, 1.

[Cao et. al, 2011] L. Cao, Y. Zheng, and Q. Zhou (2011). A necessary and sufficient condition for consensus of continuous-time agents over undirected time-varying networks. IEEE Transactions on Automatic Control, 56, 8.
[Qu, 2009], Z. Qu (2009). Cooperative Control of Dynamical Systems, Springer Verlag, London 2009.

[Martin E Girard, 2013] S. Martin and A. Girard. (2013) Continuous-time consensus under persistent connectivity and slow divergence of reciprocal interaction weights. SIAM J. Control Optim., 51, 3.

[Shi E Johansson, 2013] G. Shi and H. Johansson, (2012) The Role of Persistent Graphs in the Agreement Seeking of Social Networks, IEEE Journal of Selected areas in communications, 31, 9, 595-606.

[Lin et al., 2007] Z. Lin, B. Francis, and M. Maggiore. (2007) State agreement for continuous time coupled nonlinear systems. SIAM J. Contr., 46, 1.

[Cao \& Ren, 2011] Y. Cao and W. Ren, (2011) Distributed Multi-Agent Coordination: A Comparison Lemma Based Approach, IEEE American Control Conf., S. Francisco, CA.

[Slotine \& Wang, 2004] J.-J. E. Slotine and W. Wang, (2004) A study of synchronization and group cooperation using partial contraction theory, Springer-Verlag.

[Altafini, 2013] C. Altafini, (2013) Consensus problems on networks with antagonistic interactions, IEEE Transactions on Automatic Control, 58, 4.

[Smith, 1995] H. L. Smith. (1995) Monotone Dynamical Systems: An Introduction to the Theory of Competitive and Cooperative Systems, Volume 41 of Mathematical Surveys and Monographs. AMS, Providence, RI.

[Clarke et al., 1998$]$ F.H. Clarke, Y.S. Ledyaev, R.J. Stern and P.R. Wolenski, (1998) Nonsmooth Analysis and Control Theory, Springer, 1998. 